\title{
Nach der Reform ist vor der Reform
}

\section{Zentrale Herausforderungen und mögliche Perspektiven einer Reform der Pflegeversicherung in der kommenden Legislaturperiode}

Thomas Klie

Prof. Dr. Thomas Klie ist

Hochschullehrer für öffentliches Recht und Verwaltungswissenschaft an der Evangelischen

Fachhochschule Freiburg. Er ist Präsident der Deutschen Gesellschaft für Gerontologie und Geriatrie. E-Mail klie@efh-freiburg.de
Angesichts der demografischen Herausforderung und dem Wandel der Bereitschaft von Familien zur Pflege ibrer Angehörigen, bedarf es weitreichender Veränderungen als es die aktuelle Reform der Pflegeversicherung vorsieht. Insofern wird die Pflegepolitik auch weiterhin auf der Tagesordnung stehen.

Die Große Koalition hatte sich in der Pflegepolitik mehr vorgenommen als sie einlösen konnte. Die zentralen Fragen der Finanzierung wurde in den Wahlkampf 2009 verlagert, die Überarbeitung des Pflegebedürftigkeitsbegriffes auf Kosten der Versicherten einem Konsortium aus Wissenschaft und Medizinischem Dienst der Krankenkassen übertragen, eingebettet in einen Konsultationsprozess. Auch das Thema Pflegebudget wurde bewusst aus dem Pflege-Weiterentwicklungsgesetz (PfWG) herausgehalten. Man wollte, so hieß es, die Ergebnisse der wissenschaftlichen Begleitforschung nach Ende des Modellprojektes abwarten, allerdings das »Integrierte Trägerübergreifende Budget « in der Fläche erproben.

Damit ist bereits eine Agenda für die Reform des SGB XI in der kommenden Legislaturperiode aufgestellt. Die Abarbeitung dieser Agenda dürfte aber keinesfalls ausreichen, um die Sicherung der Pflege nachhaltig zu gewährleisten, wie dies die Überschrift für den Referenten-Entwurf zum Pflege-Weiterentwikklungsgesetz programmatisch formulierte. Die Herausforderungen reichen weiter als dies im Rahmen einer systemimmanenten Reform der Pflegeversicherung möglich ist. Insofern sind für die weiteren Legislaturperioden pflegepolitische Agenden vorauszusagen, die viel von dem was im Pflege-Weiterentwicklungsgesetz an Themen aufgegriffen wurden, in ihrem grundsätzlichen Bedeutungsgehalt erst zum Tragen bringen werden.
Die zentralen Aufgaben für eine künftige und auf Nachhaltigkeit hin angelegte Pflegepolitik lassen sich in fünf Fragen zusammenfassen:

\section{1.} Who cares? Der demografische und - soziale Wandel machen deutlich, dass die Grundlagen, auf der die Pflegeversicherung aufbaut, sowohl leistungsrechtlich als auch hinsichtlich ihrer Finanzierung in den nächsten Jahrzehnten erodieren werden. Die Pflegebereitschaft von Familien modifiziert sich. Sie verschwindet keineswegs, aber sie ist sowohl demografisch nicht mehr in gleicher Weise erwartbar als auch hinsichtlich der in der Bevölkerung verankerten Mentalitäten und Bereitschaften deutlich verändert (Abb. 1, Seite 168). Schon heute zeigen Zeitbudgetstudien, dass Menschen mit Pflegebedarf je nach sozialen Netzwerkkonstellationen und Wohnort bei gleicher Pflegebedürftigkeit mit einer höchst unterschiedlichen Intensität von Pflege und Begleitung rechnen können (Abb. 2). Die Pflegeversicherung akzentuiert viel zu stark die medizinisch pflegerische Seite und vernachlässigt die sozialen Dimensionen der Pflege - aber Pflege ist auch ein soziales Schicksal. Alle »Aufrüstung « in Richtung Professionalisierung in medizinisch pflegerischer Hinsicht geht an diesen zentralen Sachverhalten vorbei. Es geht um das »Caring «, das Sorge tragen und Kümmern für Menschen, die auf andere verwiesen sind - in der ganzen Lebensspanne. Im Care liegt eine der zentralen kulturellen Herausforderungen für die nächsten Jahrzehnte. Diese Herausforderungen enthalten weitreichende Implikationen für künftige Pflegereformen. Sie lassen sich nicht allein mit Appellen an bürgerschaftliches Engagement beantworten.

2. Wie Unterstützung und Kooperation gestalten? Wie gelingt die Unterstützung in der Lebensführung und Bewältigung bei Pflege für die Menschen 


\section{Thema}

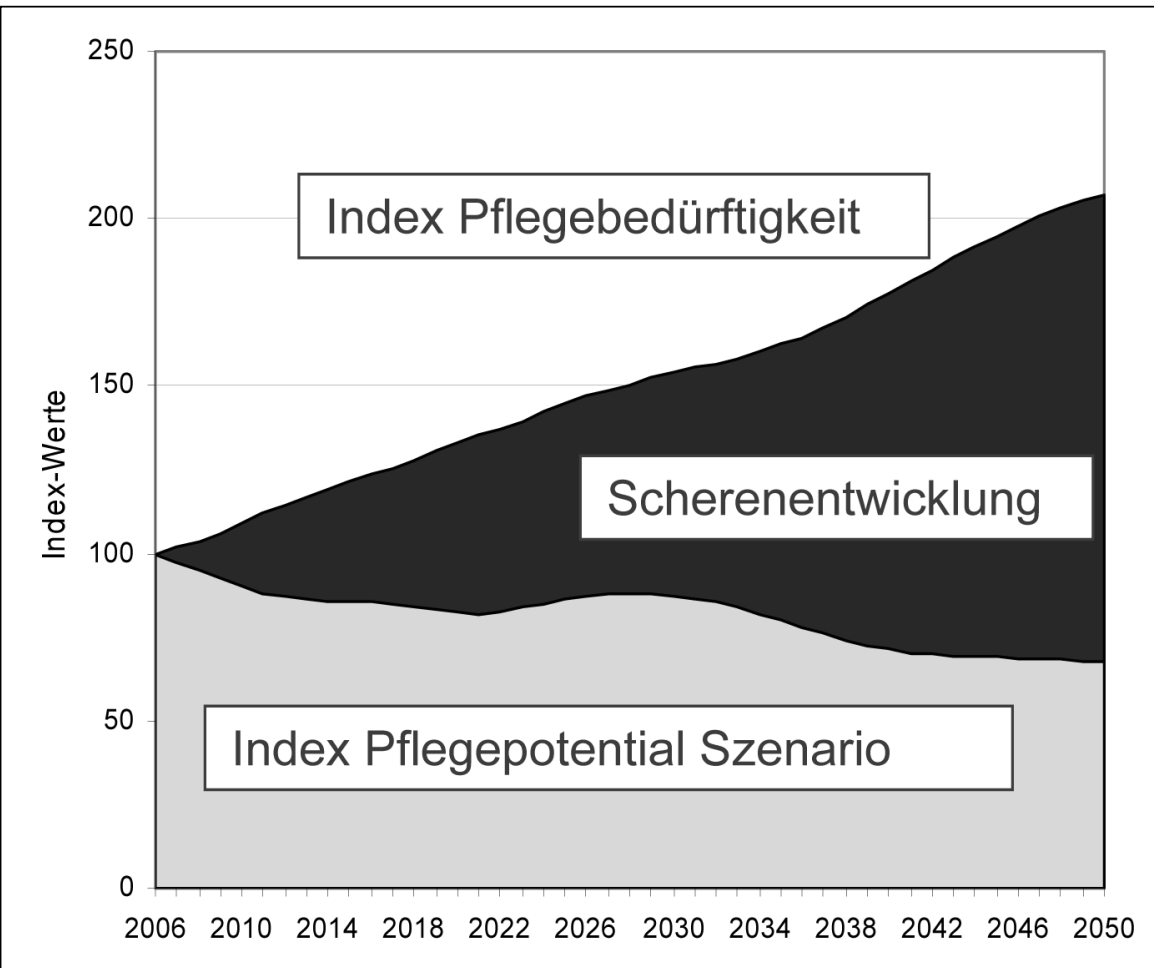

Abb. 1: Die Scherenentwicklung macht die steigende Zahl der Pflegebedürftigen und die sinkende Zahl des so genannten Pflegepotentials deutlich (Blinkert/Klie AzuP 2008, S. 27 ff.)

mit Behinderung und Pflegebedarf (eine mir angemessene erscheinende Terminologie statt des Begriffs »Pflegebedürftigkeit «) und den sie begleitenden Angehörigen? Es kann heute keineswegs befriedigen, wie sich die professionelle Begleitung von Menschen mit Behinderung und Pfle-
Weiterentwicklungsgesetz durch die Pflegeberatung und die Pflegestützpunkte den Case Management-Fachdiskurs aufgenommen, allerdings ohne die notwendigen qualifikatorischen und strukturellen Voraussetzungen für die Implementation eines wirksamen Case Managements zu schaffen. (vgl. hierzu DGCC Expertise, Internet http://www.dgcc.de). Wenn es im Sinne eines modernen Welfare-Mix (vgl. Klie/Ross 2005) gelingen soll, unterschiedliche professionelle Hilfen mit familiärer und bürgerschaftlicher Unterstützung intelligent zu verbinden und dies gerade für Personen, die nicht über hinreichende soziale Netzwerkstrukturen verfügen, dann bedarf dies einer professionellen, leistungsträger- und professionsübergreifenden Unterstützung. Die Zuordnung der Aufgaben der Pflegeberatung an die Pflegekassen greift hier nicht nur viel zu kurz sondern ist verfehlt: Case Management bedarf der lebensweltlichen und kommunalen Verankerung und nicht der Einbindung in eine zentral gesteuerte administrative Struktur.

2 Wie Teilhabe sichern? Hierzu die3 •nen medizinisch pflegerische Erkenntnisse, auch die Fachpflege, die auf einem Verständnis von Behinderung basiert, das der »Internationalen Klassifikation der Funktionsfähigkeit, Behinderung und Gesundheit « (ICF) der Weltgesundheitsorganisation folgt und auf die sozia-

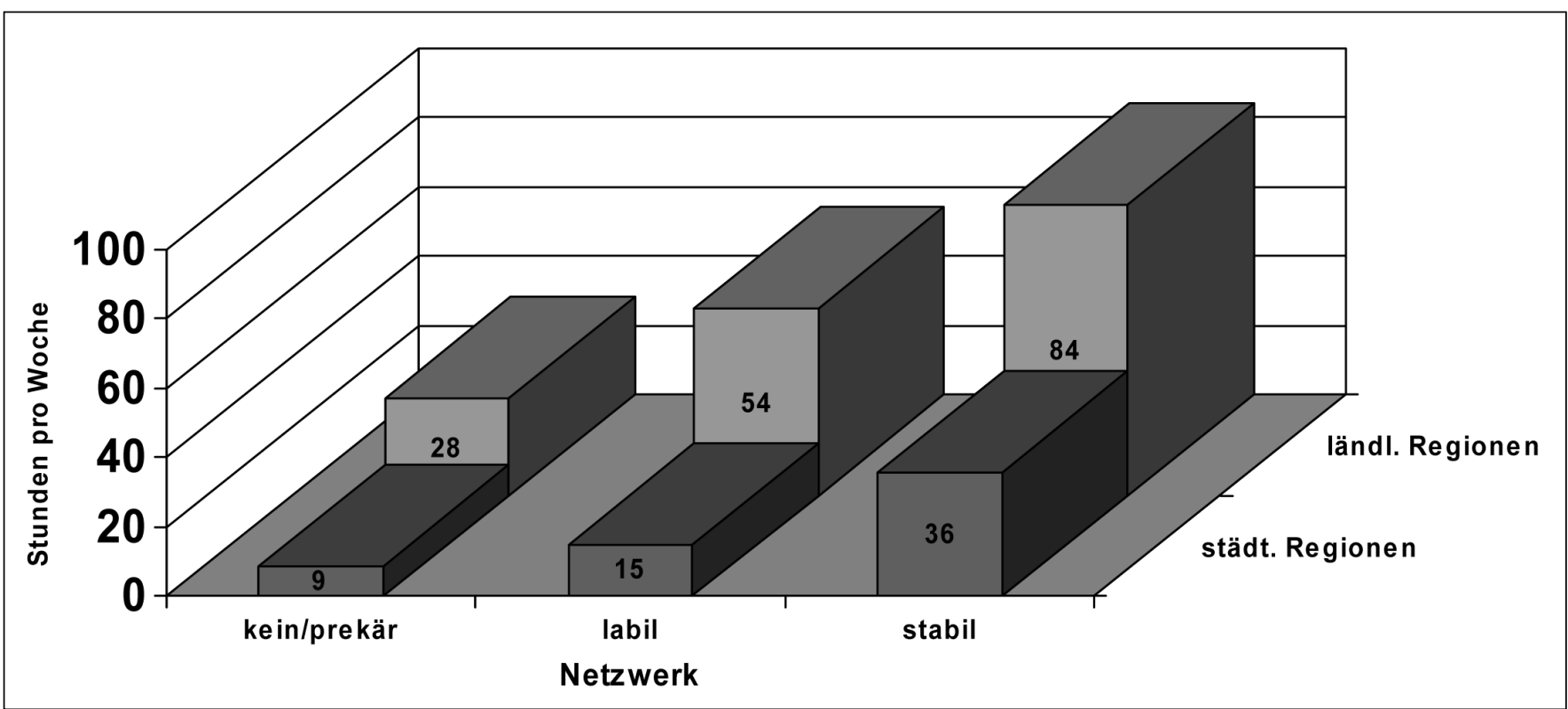

Abb. 2: Pflege als soziales Schicksal, Pflegezeit Stadt/Land: Im städtischen Umfeld lebende erhalten im Schnitt 9 Stunden pro Woche (Brutto-)Pflege- und Begleitungszeit, Menschen mit Pflegebedarf im ländlichen Bereich in soziale Netzwerkstrukturen gut integriert (meist eigene Familie) erhalten bis zu 84 Stunden. (Blinkert/Klie 2006) 
le Teilhabe von Menschen mit Pflegebedarf ausgerichtet ist. Ob im Heim oder Daheim, überall geht es um die Sicherung von Lebensqualität, um die Herstellung von Würde durch Interaktion und damit auch um die Sicherung sozialer Teilhabe. Dies ist keine Aufgabe, die allein professionellen und Institutionen übertragen werden kann, sondern die der Gesellschaft, die nur eingelöst werden kann in überschaubaren sozialen Einheiten.

4. Wie finanzieren? Im Vergleich zur - Renten- und Krankenversicherung ist die finanzielle Absicherung des Pflegerisikos eine kompliziertere Frage. Das Pflegerisiko allein einer umlagefinanzierten Finanzierungsstrategie zu überlassen, erscheint schon aus demografischen $\mathrm{Ge}$ sichtspunkten nicht nachhaltig angelegt. Man wird sich über die Fragen der Finanzierung unter unterschiedlichen Vorzeichen Gedanken machen müssen. Dabei gilt es zu reflektieren, dass Pflege mitnichten nur ein Thema ist, das sich mit klassischen Strategien in der Sachleistungslogik oder über sozialstaatliche Transferleistungen bewältigen lässt. Nimmt man zur Kenntnis, dass die bisherigen Leistungen der Pflegeversicherung, insbesondere die Sachleistungen im ambulanten Bereich, eine eher nachgeordnete Bedeutung für die Sicherung der Pflege in Privathaushalten haben, wird man an einer Neuzuschneidung von Leistungen und auch an einer Erweiterung der Leistungsformen mittelfristig nicht vorbeikommen.

5 Was macht gute Pflege aus? Schaut - man die Qualitätsberichte des Medizinischen Dienstes der Krankenkassen an, so stehen dort medizinisch pflegerische Fragen im Vordergrund - Dekubitusprophylaxe, Ernährung- und Flüssigkeitszufuhr, Pflegedokumentation, nachvollziehbare Medikamentenvergabe (vgl. MDSQualitätsbericht 2005) - und dies zu Recht. Da die medizinisch pflegerische Qualität eine wichtige Dimension guter Pflege ist, ist es überhaupt nicht hinnehmbar, dass nicht alle auf Pflege angewiesenen Menschen an dem Stand der Künste der Pflege teilhaben. Ebenso bedeutsam ist es, dass die biografisch geprägte Individualität bei der Lebensbewältigung und Lebensgestaltung unter Bedingung von Pflegebedürftigkeit berücksichtigt wird. Die Zeit- und Teilhabedimension sind bedeu- tende Qualitätsdimensionen. Ein solches Qualitätsverständnis verlangt vor allem nach subjektorientierten Instrumenten der »Qualitätssicherung «: Assessments, Hilfeplanung, Aushandlung. Viel zu eng knüpfen wir die notwendigerweise formalisier- stellen, in der Pflege funktionieren könnte. So ist mit den nächsten Reformschritten die Frage zu verbinden, wie ein neuer assessmentgestützter Pflegebedürftigkeitsbegriff, der dazu dient, alle relevanten Einflussfaktoren für die von Pflege ge-

\section{»Das Sachleistungsprinzip ist sinnvoll, wo professionelle Standards in der Versorgung sicherzustellen sind"}

te Definition von Pflegebedürftigkeit als Leistungsvoraussetzung für SGB XI Leistungen an Inhalte der Leistungen an, die dann einer strengen Qualitätskontrolle unterworfen werden. Das Gerechtigkeitsproblem in der Verteilung knapper Mittel wird im SGB XI »unterkomplex « gelöst.

\section{Perspektiven}

Aus den Hinweisen auf die zentralen Herausforderungen lassen sich auch mögliche Perspektiven für eine angemessene Pflegepolitik ableiten:

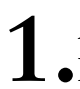

Assessment, Begutachtungs- und -Hilfeplanung integrieren: Es liegt eine große Chance in der Überarbeitung des Begutachtungsverfahrens im SGB XI und des Pflegebedürftigkeitsbegriffes, wenn es gelingt, fachlich bedeutsame Indikatoren für die gesundheitliche Situation von auf Pflege angewiesenen Menschen und ihrer Erhebung mit einer gemeinsamen Inblicknahme der Lebenssituation und dem Aushandlungsprozess mit allen Beteiligten, wie die Lebensbewältigung angesichts der Pflegeaufgaben geschehen soll, zu verbinden. Es ist gesundheitsökonomisch nicht sinnvoll, Begutachtungsverfahren von fachlicher Assessment- und Hilfeplanungsfunktion zu trennen. Gelingt es einen assessmentgestützten Pflegebegriff zu etablieren, mit validen und reliablen Instrumenten, sollte dessen Anwendung allen Profis möglich sein, so dass es für die Feststellung der Pflegebedürftigkeit, außer einer supervisorischen Funktion nicht mehr bedarf. Verfahrensrechtlich bietet das SGB IX ein Muster, das bei entsprechender Ressourcenzuordnung anders als bei den Service- prägte Lebenssituation in den Blick zu nehmen, dem Leistungsrecht, etwa im SGB I, vorangestellt werden kann. Er wäre dann für alle Leistungsträger verbindlich und würde auch auf struktureller Ebene neue Gestaltungsoptionen für die Begutachtungsassessment- und Planungsfunktion eröffnen.

2. Strukturreformen: Eine Strukturreform der Pflegeversicherung wäre damit verbunden, die das, was im PflegeWeiterentwicklungsgesetz nicht nur halbherzig, sondern so geregelt wurde, dass ordnungspolitisch betrachtet keine relevanten Strukturen entstehen können, die eine neue Bedeutung der kommunalen Ebene und ein neues Miteinander von sektorübergreifenden Akteuren etabliert.

2 Neuordnung des Leistungsrechts: 3 •Inzwischen ist vergleichsweise viel über die Qualität von Pflegeleistungen sowohl im ambulanten als auch im stationären Bereich bekannt - vergleichsweise wenig über die Effizienz der Leistungen im Zusammenhang mit der Bewältigung der Lebenssituation der Betroffenen, gleiches gilt für die Akzeptanz des Pflegegeldes und über seine Verwendung und seine Wirkungen. Das Leistungsrecht des SGB XI ist noch strikt eingebunden in eine Sachleistungslogik, verbunden mit einem verrichtungsbezogenen, protonormistischen Pflegebedürftigkeitsbegriff. Insofern wird man sich in der nächsten Legislaturperiode auch über die Leistungsformen Gedanken machen - dazu gehört das Pflegegeld. Wir kennen heute drei unterschiedliche Verwendungsarten: Stützung der Familienpflegebereitschaft, Nutzung i.S. der Grundsicherung/Lebensstandardsicherung und den Einsatz für 
Schwarzarbeit. Die Freiheit, die das Pflegegeld gewährt ist bestechend. Gleichwohl sollte man überlegen, ob man die Vereinbarkeitspolitik nicht stärker durch ein zweckbestimmtes Pflegegeld stützt und das Pflegegeld wirklich auf eine Gratifikationsfunktion begrenzt, wie es das Kindergeld darstellt. Die Pflegezeit mit einer Einkommensersatzleistung zu flankieren ist familien- und vereinbarkeitspolitisch von hohem Belang. Auch die Sachleistungen sollten überdacht werden. Das Prinzip der Sachleistung macht vor allen Dingen dort Sinn, wo es darum geht, professionelle Standards in der Versorgung sicherzustellen. $\mathrm{Zu}$ den professionellen Pflegeaufgaben gehören Steuerungsfunktionen und begrenzt besonders anspruchsvolle »handwerkliche « Aufgaben. Diese fachpflegerischen Leistungen ließen sich ggf. in das GKV-System integrieren.

\section{Schlussbemerkung}

Das Pflege-Weiterentwicklungsgesetz gibt wichtige Impulse, signalisiert die Bereitschaft der Politik, sich des Themas auch mit etwas mehr Geld anzunehmen und thematisiert Grundfragen zukünftiger Pflegesicherung.

Die Stärkung der Leistungsträger Pflegekassen und das Beharren in korporatistischen Strukturen wird den Herausforderungen, die sich angesichts des demografischen und sozialen Wandels stellen, allerdings nicht gerecht. Die zentralen Fragen der Finanzierung und des Pflegebegriffes wurden vertagt. Insofern wird in der nächsten Legislaturperiode die Pflegereform prominent auf der politischen Agenda stehen.

Die beschriebenen Herausforderungen und skizzierten Perspektiven legen eine grundlegende Reform der Pflegesicherung nahe: Ein neues Pflegeverständnis im Sinne von Care zu etablieren und eine Strukturreform mit einer neuen und symmetrischeren Aufgabenverteilung zwischen Bund, Ländern, Kommunen und eine neue Architektur des Leistungsrechts.

Nicht mehr vom Selben sondern Neues ist gefragt, wenn es um die Sicherung der Pflege geht. Das Erfolgsmodell Pflegeversicherung wird seine Bewährungsprobe erst dann bestehen, wenn sie sich ebenso wie die Arbeitsverwaltung - in grundlegender Weise als reformfähig erweist.

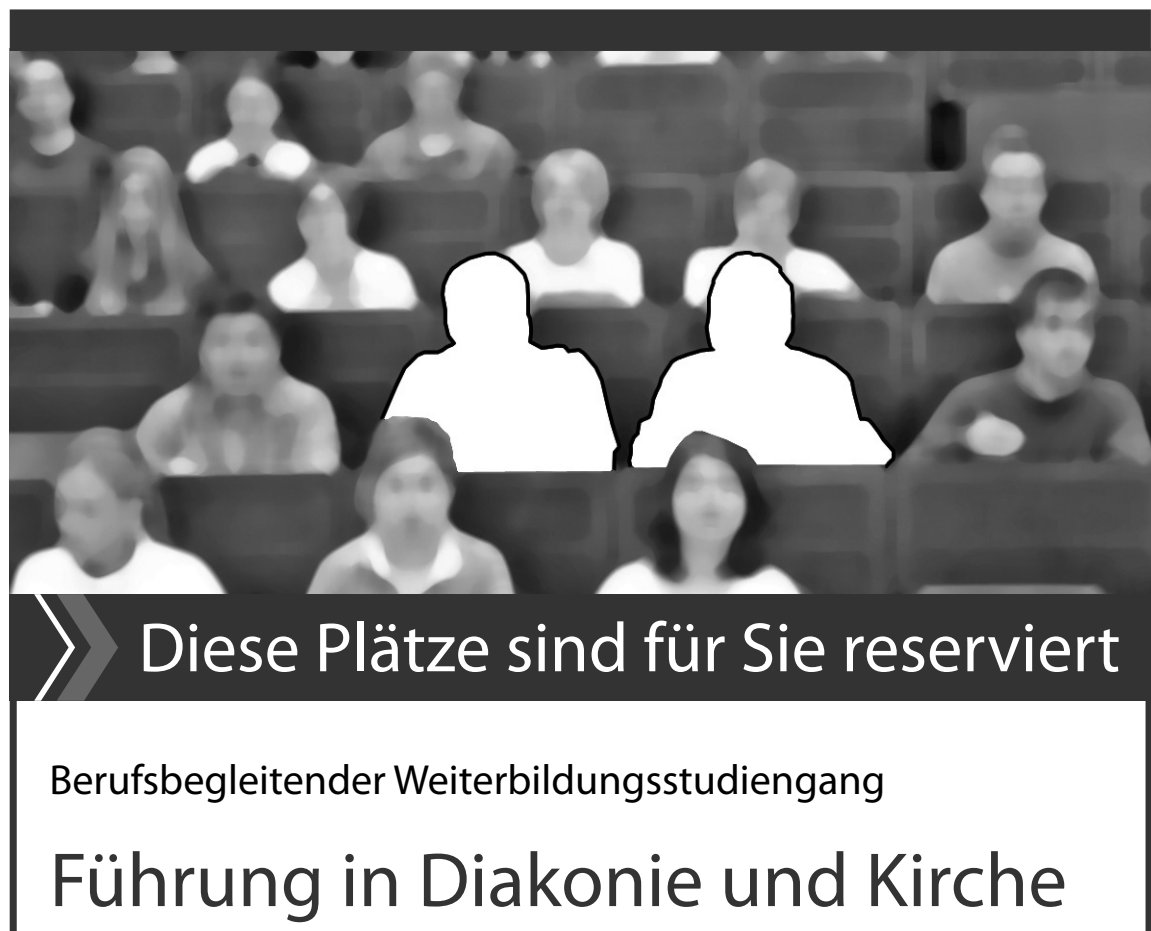

\section{Masterstudiengang Diakoniewissenschaft}

\author{
Mit dem Abschluss Master of Arts
}

Ruprecht-Karls-Universität Heidelberg - Diakoniewissenschaftliches Institut und die EFH in Darmstadt, Freiburg und Reutlingen-Ludwigsburg Kontakt: Ilona Agoston, Tel. 062 21/54-81 57 (Mo. und Do.)

masterstudiengang@dwi.uni-heidelberg.de

http://www.dwi.uni-hd.de/studium 